\title{
Temporal bone fractures: a clinical diagnosis
}

\author{
J. WALDRON \& S. E. J. HURLEY
}

Department of Ear Nose and Throat Surgery, St Mary's Hospital, London, England

\section{SUMMARY}

Temporal bone fractures may be complicated by intracranial haemorrhage, C.S.F. $\cong$ leakage and infection, damage to the middle and inner ear and damage to the seventh and eighth cranial nerves. Accurate early diagnosis is important to enable adequate investigation and prompt treatment of any complications.

We present eight cases seen in a 12 month period in which a temporal bone fracture was not diagnosed at presentation in spite of a full clinical examination and standard skull radiographs. Five of these cases developed complications which resulted in the $\overrightarrow{8}$ referral.

The absence of a visible fracture on plain skull radiographs does not exclude $\frac{a}{7}$ fracture, and those patients with clinical signs of a fracture should be treated appropriately and further investigations performed. Therefore the clinical examination is vital in diagnosing temporal bone fractures and must include careful otoscopy together with assessment of the function of the seventh and eighth cranial nerves.

\section{INTRODUCTION}

The petrous part of the temporal bone contains the structures of the middle and inner ear, and the seventh and eighth cranial nerves, which renders these vulnerable to injury when the bone is fractured. The floor of the middle cranial fossa over the region of the middle ear and mastoid, the tegmen tympani, is a thin bony plate, and disruption of this can produce a dural tear and leak of C.S.F. into the ear. This results in C.S.F. otorrhoea, or C.S.F. rhinorrhoea via the Eustachian tube, if the tympanic membrane is intact.

Fractures of the petrous part of the temporal bone are commonly divided into longitudinal and transverse types, although high resolution CAT scanning and surgical exploration have shown that these fractures are often complex (Adour et al., 1977).

Correspondence: Dr S.E.F. Hurley, Department of Ear Nose and Throat Surgery, St Mary's Hospital, Praed Street, London W2 INY, England. 
Longitudinal fractures account for approximately 70-80\% (Hough \& Stuart, 1968). These fractures parallel the long axis of the temporal bone, and frequently involve the roof of the external auditory meatus running through the middle ear, but usually sparing the inner ear (Cannon \& Jahrsdoerfer, 1983). Examination of these patients may reveal disruption of the roof of the external auditory meatus, a haemotympanum, a C.S.F. leak, and a conductive hearing loss, due either to blood or C.S.F. in the middle ear or to ossicular discontinuity. Sensorineural hearing loss is uncommon in these injuries as is damage to the facial nerve which is seen in $10-20 \%$ of cases and is usually delayed (Lindeman, 1979).

Transverse fractures usually run medial to the middle ear through the inner ear (Wiet et al., 1985). In these cases otoscopy may not reveal any abnormality, but a sensorineural hearing loss, which may be total, is found in up to $50 \%$ (Cannon \& Jahrsdoerfer, 1983). Facial nerve paralysis, which may be total, is found in $40-50 \%$ (Lindeman, 1979). Those patients with damage to the inner ear or eighth nerve are almost always acutely vertiginous with obvious nystagmus.

All patients with skull fractures should be observed in hospital initially, as they are at a significant risk of developing an intracranial haematoma (A Group of Neurosurgeons, 1984). In addition, patients with a fracture of the temporal bone, which comprise $22 \%$ of skull fractures sustained (Cannon \& Jahrsdoerfer, 1983), are at risk of other complications described above, which may require further investigation and management.

Temporal bone fractures are seldom displaced, and the fracture line may be obscured by superimposed bony structures on conventional radiography. They are therefore difficult to demonstrate on routine skull films (Cannon \& Jahrsdoerfer, 1983). The following cases referred to our department over a 12 month period illustrate the importance of the clinical examination in diagnosing temporal bone fractures.

\section{PATIENTS}

Eight patients referred to our department for further assessment following a head injury over a 12 month period are presented. All of the patients were male, aged between 19 and 60 years. These patients' details are summarised in Table I.

Six of the patients presented to the A\&E department immediately after their injury. Two presented with delays of 24 and 36 hours respectively. Six of the patients were admitted immediately for observation with a diagnosis of head injury. In three of these a skull fracture was seen on plain radiographs, but none involved the temporal bone (one occipital and two parietal fractures).

One patient had a partial facial palsy at presentation. All patients underwent otoscopy at presentation which was recorded as abnormal in five cases; three had a bloody discharge and two had clear fluid in the external auditory meatus. Nystagmus was noted in the $A \& E$ department in two of these cases, in one of which it was considered to be secondary to alcohol intoxication.

The patients were referred to us for assessment immediately (three cases) or within 72 
Table 1 Findings in eight patients

\begin{tabular}{ll}
\hline At presentation & \\
Immediate presentation to A\&E Dept. & 6 \\
Skull X-rays performed & 6 \\
Temporal bone fracture seen & 0 \\
Other skull fracture seen & 3 \\
Admission to hospital & 6 \\
VII nerve palsy & 1 \\
Abnormal otoscopy in A\&E & 5 \\
& \\
At referral & \\
C.S.F. leak & 5 \\
Nystagmus & 4 \\
Otoscopy abnormal & 8 \\
Tuning fork tests abnormal & 8 \\
Pure tone audiogram abnormal & 8 \\
Further radiology + ve & $5 / 5$ \\
VII nerve palsy-delayed & 4 \\
\hline
\end{tabular}

hours (five cases). Clinical examination was abnormal, and diagnostic of a temporal bone fracture in all cases.

Four patients had nystagmus when carefully examined, and one had a partial facial $\overrightarrow{0}$ palsy on the affected side. Otoscopy was abnormal in all eight cases; C.S.F. leakage wigigo seen in five patients, and a torn tympanic membrane or haemotympanum in the othơr three. A step deformity of the roof of the external auditory meatus was seen in three cases.

Tuning fork tests were abnormal in all eight cases; a conductive hearing loss shown⿳亠丷厂

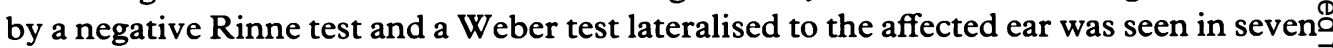
cases; a sensorineural hearing loss, shown by a positive Rinne test and a Weber testō lateralised to the non-affected ear was seen in one case. Audiology confirmed the findings in all cases. Figure 1 shows an audiogram demonstrating a high-tone sensori $-\vec{F}$ neural hearing loss due to inner ear trauma, with a superimposed low-tone conductive loss due to C.S.F. in the middle ear.

Further radiological investigation demonstrating the temporal bone fracture was performed in five cases; four of the cases had a high resolution CAT scan, and one ai hypocycloidal polytomogram.

In addition to the patient with an immediate-onset facial palsy, four other patientso developed delayed-onset facial palsies. These appeared between 12 hours and 7 days after the injury, two were complete palsies. All of these patients were followed up withㅡ.. electromyography, and all had complete resolution of the palsy within 9 months. None of these cases required surgical decompression of the facial nerve.

None of the patients in this group developed intracranial sepsis, however, all of themN were treated with prophylactic antibiotics after the fracture was diagnosed (penicillin Vo $500 \mathrm{mg}$ qds and sulphadimidine $500 \mathrm{mg}$ qds). All of the C.S.F. leaks resolved spontato neously within six weeks. None of the patients developed any other neurologica依 complication as a result of their head injuries. 


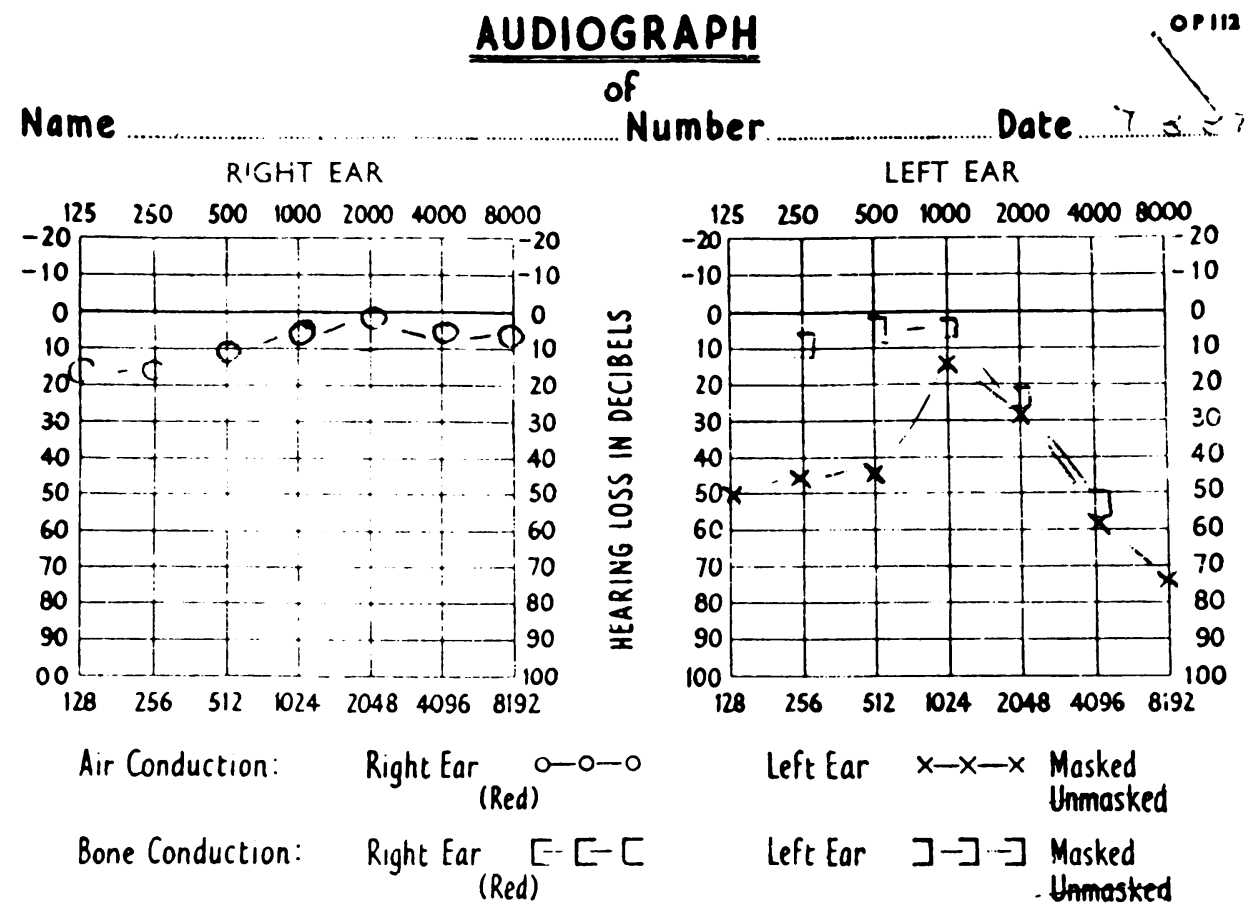

Fig. 1 Audiogram demonstrating high-tone sensorineural hearing loss with superimposed low-tone conductive loss.

\section{DISCUSSION}

Temporal bone fractures require prophylaxis against infection, and complications may require surgical or medical treatment (facial nerve palsy, persistent C.S.F. leak, conductive hearing loss). It is important, therefore, to make the diagnosis of temporal bone fracture promptly, and to assess the patient carefully for evidence of these complications. In the eight cases described above none had a visible temporal bone fracture on plain radiographs although three had evidence of other skull fractures.

The absence of a fracture on the plain radiographs made those responsible for the patients' primary care reluctant to make a definite diagnosis of temporal bone fracture despite abnormal findings on otoscopy in five of the cases. However, clinical examination of the ear (otoscopy together with tuning fork tests) showed the signs of temporal bone fracture in all cases.

These cases illustrate the limited value of plain radiographs in showing fractures of the petrous temporal bone (Lindeman, 1979). It is vital that patients with head injuries undergo careful clinical examination, which includes, as well as otoscopy, tuning fork testing. If, in the absence of direct trauma to the ear, blood or C.S.F. is seen in the external auditory meatus or a haemotympanum is present, then a diagnosis of temporal bone fracture should be made. Blood in the external auditory meatus should only be 
removed gently, under direct vision, using aseptic techniques, if further visualisation of the damage is necessary.

Tuning fork tests are necessary to confirm the resulting hearing loss, remembering that otoscopy is not always abnormal in cases of transverse temporal bone fracture.

The patient should be admitted to hospital for a period of observation. During this time further assessment can be made and this should include an audiogram in all cases. Electromyography and electroneuronography should be performed where there are signs of facial nerve injury (Fisch, 1974). All patients should be given prophylactic antibiotics and the affected ear should be left unpacked except in the event of lifethreatening haemorrhage (Cannon \& Jahrsdoerfer, 1983).

Further radiology should be performed where the complications of facial nerve $\vec{\omega}$ damage or C.S.F. leak are seen (Wiet et al., 1985) and in any cases with a deterioration $\frac{\mathscr{D}}{\mathbb{D}}$ in the level of consciousness or the development of focal neurological signs (A Group of 3 . Neurosurgeons, 1984). A high resolution CAT scan is the investigation of choice. $\stackrel{v}{\omega}$ Intrathecal metrizamide contrast can be used to determine the site of a C.S.F. leak if it $\overrightarrow{\vec{\sigma}}$ fails to resolve spontaneously and surgical repair is being considered.

\section{ACKNOWLEDGEMENTS}

We thank Mr R. Touquet, Consultant in A\&E Medicine, for his help in the preparation of this paper, also Mr G. Walsh-Waring and Mr J. Wright for permitting us to prese details of patients under their care.

\section{REFERENCES}

A Group of Neurosurgeons (1984) Guidelines for initial management after head injury in adults. British Medical fournal 288, 983-5.

Adour K. K., Boyajian J. A., Kahn Z. M. \& Schneider G. S. (1977) Surgical and nonsurgical management of facial paralysis following closed head injury. Laryngoscope 87, 380-90.

Cannon C. R. \& Jahrsdoerfer R. A. (1983) Temporal bone fractures. Review of 90 cases. Archives of Otolaryngology 109, 285-8.

Fisch U. (1974) Facial paralysis in fractures of the petrous bone. Laryngoscope 84, 2141-54.

Hough J. V. D. \& Stuart W. D. (1968) Middle ear injuries in skull trauma. Laryngoscope 78, 899-937.

Lindeman R. C. (1979) Temporal bone trauma and facial paralysis. Otolaryngologic Clinics of North America 12, 403-13.

Wiet R. J., Valvassori G. E., Kotsanis C. A. \& Parahy C. (1985) Temporal bone fractures. State of the art review. The American fournal of Otology 6, 207-15. 